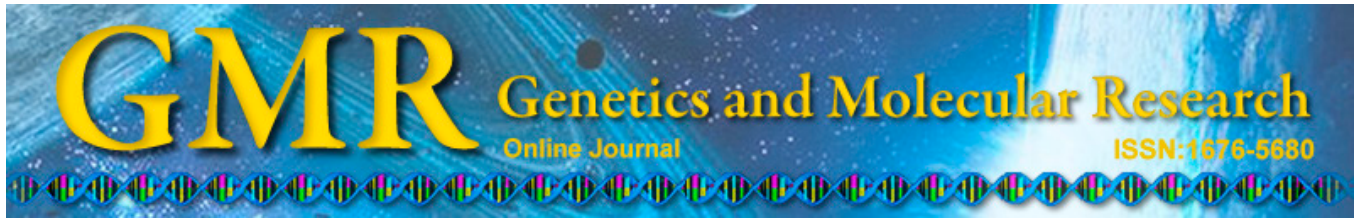

\title{
Quantitative real-time polymerase chain reaction as an efficient molecular tool for detecting minimal residual disease in Moroccan chronic myeloid leukemia patients
}

\author{
A. Moumen ${ }^{1 *}$, H. Dehbi ${ }^{2 *}$, D. Kottwitz ${ }^{1}$, M. El Amrani ${ }^{1}$, \\ N. Bouchoutrouch ${ }^{1}$, H. El Hadi' ${ }^{1}$, A. Quessar ${ }^{3}$, S. Benchekroun ${ }^{3}$, \\ S. Nadifi ${ }^{2}$ and H. Sefrioui ${ }^{1}$ \\ ${ }^{1}$ Moroccan Foundation for Advanced Science, \\ Innovation and Research Rabat, Morocco \\ ${ }^{2}$ Laboratory of Human Genetics, Faculty of Medicine, Casablanca, Morocco \\ ${ }^{3}$ Hematology Department, Ibn Rochd University Hospital, Casablanca, Morocco \\ *These authors contributed equally to this study. \\ Corresponding author: H. Sefrioui \\ E-mail: h.sefrioui@mascir.com
}

Genet. Mol. Res. 14 (1): 1044-1055 (2015)

Received January 22, 2014

Accepted June 18, 2014

Published February 6, 2015

DOI http://dx.doi.org/10.4238/2015.February.6.8

\begin{abstract}
Chronic myeloid leukemia (CML) is characterized by $B C R-A B L$ translocation and an increased number and migration of immature myeloid cells into the peripheral blood. The detection limit of the $B C R-A B L$ transcript, particularly after treatment, is controversial. In the present study, we used quantitative real-time reverse transcription-polymerase chain reaction (RT-qPCR) to monitor $B C R-A B L$ expression in Moroccan CML patients undergoing imatinib treatment, and compared the results with those of conventional PCR and fluorescence in situ hybridization (FISH). The aim of this study was to establish a new molecular tool for in vitro diagnosis of CML. In a retrospective comparative analysis, 20 CML Moroccan patients
\end{abstract}


who had received imatinib treatment $(\mathrm{N}=20)$ were analyzed by realtime PCR, conventional RT-PCR, and FISH. Half of the samples analyzed $(\mathrm{N}=10)$ were positive for $B C R-A B L$ gene expression, while the other half $(\mathrm{N}=10)$ were negative according to conventional PCR. Interestingly, 5 of the 10 samples shown to be negative by conventional PCR showed positive expression of the $B C R-A B L$ gene according to RT-qPCR. The RT-qPCR results were confirmed by FISH, which revealed a high concordance $(100 \%)$ rate. We found that real-time RTqPCR is more reliable and should be used in Moroccan biomedical analysis laboratories to monitor CML progression, particularly for minimal residual disease, following imatinib treatment.

Key words: Chronic myeloid leukemia; BCR-ABL; Imatinib treatment; Molecular diagnostics; Moroccan chronic myeloid leukemia patients;

Quantitative real-time polymerase chain reaction

\section{INTRODUCTION}

Chronic myelogenous leukemia (CML) is a hematopoietic stem cell disorder with an estimated annual occurrence of 1-2 cases per 100,000 individuals per year (Sawyers, 1999). The clinical path in CML is generally divided into a chronic phase (CP), accelerated phase, and blast phase (Faderl et al., 1999). While CP is characterized by massive expansion of myeloid cells that maintain normal maturation, the accelerated and blast phases are featured by leukemic cells that lose their capacity to terminally differentiate and give rise to acute leukemia, which is highly refractory to therapy (Faderl et al., 1999). The causative molecular defect is the BCR-ABL protein encoded by the Philadelphia chromosome (Ph) (Rowley, 1973; Faderl et al., 1999). This genetic abnormality is a result of an exchange of genetic material between chromosomes 9 and 22, giving rise to a fusion of the breakpoint cluster region $(B C R)$ and the c-ABL proto-oncogene protein found in the Abelson murine leukemia virus (v-abl) (Rowley, 1973; Heisterkamp et al., 1985). c- $a b l$ encodes for a non-receptor tyrosine kinase with tightly regulated kinase activity and shuttles between the nucleus and cytoplasm, whereas the BCR-ABL fusion proteins are exclusively cytoplasmic and show enhanced tyrosine kinase activity that is essential for their transforming ability (Lugo et al., 1990; Sillaber et al., 2003; Sattler and Griffin, 2003). Indeed, transduction of $B C R-A B L$ into murine hematopoietic stem cells followed by transplantation into irradiated mice produces a CML-like syndrome, implicating $B C R-A B L$ as the causative molecular abnormality of CML (Daley et al., 1990). Depending on the breakpoint site in $B C R$, different fusion proteins are produced, including p185 (185 kDa), p210 (210 kDa), or, rarely, $\mathrm{p} 230$, which respectively correspond to $\mathrm{m}, \mathrm{M}$, and $\mathrm{uBCR}-\mathrm{abl}$. While the $\mathrm{p} 210$ protein is observed in $95 \%$ of patients with CML, the other protein forms are found in other forms of acute leukemia such as acute lymphoblastic leukemia (Faderl et al., 1999).

Intensive effort has been exerted to identify a specific inhibitor of BCR-ABL tyrosine kinase. This has resulted in the identification of the specific inhibitor imatinibmesylate (STI571), which has now become the standard first-line therapy for patients with CP-CML (Savage and Antman, 2002; Capdeville et al., 2002). International randomized studies of interferon and STI571 (IRIS) have provided clear evidence of the superiority of imatinib over the previous choice of drug therapy such as interferon- $\alpha$ and cytarabine (Lyseng-Williamson 
and Jarvis, 2001; Savage and Antman, 2002; Capdeville et al., 2002). Imatinib efficacy was defined by measuring $B C R-A B L$ transcript levels to evaluate the minimal residual disease (MRD) load in patients who had reached a complete cytogenetic response (CCyR) $(0 \% \mathrm{Ph}$ positive chromosome in 20 metaphases) (Hughes et al., 2003). A decrease of more than $3 \log$ in $B C R-A B L$ fusion transcript levels from the baseline mean, defined as a major molecular response, is a favorable prognostic factor for the disease at any time during therapy. In terms of treatment responses, it has been reported that this BCR-ABL kinase inhibitor produces a complete hematological response in $98 \%$ of patients, whereas a CCyR occurs by 60 -month follow-up in $87 \%$ of newly diagnosed patients with CML in CP, as well as $40-60 \%$ of patients in late-stage CP (Druker et al., 2001; Kantarjian et al., 2002). Patients who did not achieve a complete hematological response at 3 months or CCyR at 18 months were at an increased risk of relapse (Kantarjian et al., 2002). Despite this breakthrough in treating CML patients, up to $27 \%$ of patients who achieved CCyR subsequently lost their response and consequently failed to gain a sufficient or permanent clinical benefit because of intolerance and/or resistance (Wang et al., 2001; Kantarjian et al., 2002). An increase in $B C R-A B L$ transcript levels detected during imatinib therapy for Ph-positive CML patients who achieved CCyR is an alarming indicator of suboptimal response and should trigger a subsequent, more stringent assessment method (Kantarjian et al., 2002). Both conventional karyotyping and fluorescence in situ hybridization (FISH) are the classical methods used either to detect Ph-positive cells or to estimate the level of these cells to monitor the disease response to treatment (Buño et al., 1998; Wang et al., 2001). However, both the accuracy and sensitivity of these methods, mainly in patients with CCyR, remains controversial (Buño et al., 1998; Wang et al., 2001). Therefore, molecular monitoring of the $B C R-A B L$ transcript levels by quantitative reverse transcriptionpolymerase chain reaction (RT-qPCR) during treatment may be the most efficient tool for evaluating the outcome and assessing the risk of relapse. RT-qPCR for $B C R-A B L$ constitutes the backbone of CML monitoring in various centers worldwide. Currently, in Morocco, most laboratories use classical RT-PCR and the expensive FISH method to monitor CML treatment response and to detect MRD. Thus, we investigated whether serial supervision of $B C R-A B L$ expression by RT-qPCR is more beneficial for the molecular monitoring of CML and whether this method allows for accurate measurement of MRD in Moroccan patients.

\section{MATERIAL AND METHODS}

\section{Patients}

Twenty CML patients (10 males, 10 females) treated with imatinib underwent molecular follow-up in our institute after CCyR. Patients were recruited at the University Hospital of Casablanca, where $B C R-A B L$ translocation detection was performed by FISH and conventional RT-PCR. The results were analyzed retrospectively. Mean age at diagnosis was 51 years (range 23-72 years). Mean disease duration before imatinib therapy was 3.3 years (range 0.111.8 years). Thirteen patients were treated in the CP phase, 4 patients in the accelerated phase, and 3 patients in blastic crisis. Twelve patients in CP received imatinib for interferon-resistant disease and 5 patients received it because of intolerance to interferon. Patients received 400 $\mathrm{mg}$ imatinib daily for CP-CML and $600 \mathrm{mg}$ daily for accelerated phase and blast phase CML, with the option of dose adjustment according to the clinical course. The median follow-up from the initiation of imatinib to the last test obtained was 14.9 months (range 1-30 months). 


\section{Isolation of peripheral blood mononuclear cells (PBMCs) from patient blood samples}

Five milliliters fresh peripheral blood from each patient was collected and mixed with $10 \mathrm{~mL}$ Hanks' buffered salt solution. This blood mixture was gently placed in a $40-\mathrm{mL}$ tube containing $5 \mathrm{~mL}$ Ficoll and centrifuged at $558 \mathrm{~g}(1600 \mathrm{rpm})$ or $30 \mathrm{~min}$ at $4^{\circ} \mathrm{C}$. After discarding the upper plasma layer, the interface layer containing PBMCs was gently collected in a sterile 50-mL tube, which was washed twice with Hanks' buffer. The cells were counted and stored in dimethyl sulfoxide and fetal calf serum at $-80^{\circ} \mathrm{C}$.

\section{RNA isolation and reverse transcription}

\section{RNA extraction}

Total RNA extraction was performed using the RNeasy Mini kit from Qiagen (Hilden, Germany) according to manufacturer instructions. The concentration of purified RNA was determined using the Nanodrop system (Thermo Scientific, Waltham, MA, USA) and the integrity of this nucleic acid was analyzed using the Bioanalyzer (Bio-Rad, Hercules, CA, USA). RNA was stored at $-80^{\circ} \mathrm{C}$ until use.

\section{cDNA synthesis}

The reaction was performed in a total volume of $20 \mu \mathrm{L}$ using the High-Capacity cDNA Reverse Transcription Kit (Applied Biosystems, Foster City, CA, USA), and up to $2 \mu \mathrm{g}$ RNA was used for the RT reaction following manufacturer instructions. The reaction mixture was incubated at $25^{\circ} \mathrm{C}$ for 10 min followed by 2 incubation periods at $37^{\circ} \mathrm{C}$ for $60 \mathrm{~min}$; the reaction was stopped by incubation at $84^{\circ} \mathrm{C}$ for $30 \mathrm{~s}$. The cDNA was stored at $-20^{\circ} \mathrm{C}$.

\section{Preparation of quantitation standards}

Standards were prepared by cloning PCR products using the cDNA of $B C R-A B L$ (e14a2) and normal $A B L$ as a template. These clones constitute stable standards for long-term use and storage. The standards had identical nucleotide sequences to their respective targets. The standards were prepared by extracting RNA from the K562 cell line. Next, $2 \mu \mathrm{g}$ RNA was reverse-transcribed and the cDNA was amplified using PCR primers, producing fragments that were slightly larger than the amplicons of the quantitative assay. The primers for preparing the standards are available upon request. The PCR product was gel-purified using the QIAquick ${ }^{\circledR}$ Gel Extraction kit (Qiagen) according to manufacturer instructions. The purified PCR product was cloned using the PromegapGEM-T Vector System (Promega, Madison, WI, USA) following manufacturer instructions. The recombinant plasmid DNA was isolated and purified using the QIAprep Spin Miniprep kit (Qiagen). Plasmid concentration was determined by measuring the absorbance at $260 \mathrm{~nm}$ using the Nanodrop 2000 system (Thermo Scientific) and the copy number calculated using the molecular weight of the plasmid. Serial dilutions of each standard were prepared in the range from $10^{6}$ to $10^{1}$ copies per $50 \mu \mathrm{L}$ in $1 \mathrm{ng} /$ $\mu \mathrm{L}$ genomic DNA extracted from the K562 cell line. 


\section{Real-time PCR}

The Primer 3 software (http://simgene.com/Primer3) was used to design appropriate fluorescent hybridization probes and primer pairs for quantification. The probes were synthesized by Eurofins with a 5' 6-carboxyfluorescein (FAM) for $B C R-A B L$ and 5' 4,7, 2'-trichloro-7'-phenyl-6-carboxyfluorescein (VIC) for $A B L$ as reporter dyes, as well as a 3' Carboxytetramethylrhodamine (TAMRA) as a quencher dye for both probes. The primers for $B C R-A B L$ were designed to amplify both the e $13 \mathrm{a} 2$ and the e $14 \mathrm{a} 2$ variants. The $A B L$ primers amplified a sequence that was disrupted during formation of the $B C R-A B L$ gene and was present in both isoforms a and $\mathrm{b}$ of the $A B L$ transcripts. The primer design also excluded amplification of genomic DNA. The sequence of the primers and probes used in the study were: $B C R-A B L$ forward: TCCGCTGACCATCAATAAGG; $B C R-A B L$ reverse: CCCTGAGGCTCAAAGTCA GA; $B C R-A B L$ probe: CCCTTCAGCGGCCAGTAGCA; $A B L$ forward: TGGAGATAACACT CTAAGCATAACTAAAGGT; $A B L$ reverse: GATGTAGTTGCTTGGGACCCA; and $A B L$ probe: AAATGGCCAAGGCTGGGTCC.

Amplification was performed in the ABI/Fste 7500 Real-Time PCR System (Applied Biosystems, Foster City, CA, USA) using TaqMan ${ }^{\circledR}$ Fast Universal PCR Master Mix (Applied Biosystems), including $2 \mathrm{X}$ concentrations of all reaction components necessary for the $5^{\prime} \mathrm{nu}-$ clease assay, except the primers and probe. The universal mix Amplitaq Gold was used for a hot-start PCR. The PCR components and the PCR cycler conditions were identical for the 3 transcripts, allowing all 3 reactions to occur on the same 96-well reaction tray. The PCR mix contained $12.5 \mu \mathrm{L}$ Universal Master Mix, $300 \mathrm{nM}$ both primers, and $200 \mathrm{nM}$ probe in a total volume of $25 \mu \mathrm{L}$. The $6 \log$ series of standards were processed with every PCR. A total of $5 \mu \mathrm{L}$ cDNA (25 ng) or standard was added to $20 \mu \mathrm{L}$ PCR Mix. The PCR cycler conditions were $95^{\circ} \mathrm{C}$ for $20 \mathrm{~s}, 50$ cycles at $95^{\circ} \mathrm{C}$ for $1 \mathrm{~s}$, and a combined annealing and extension step at $60^{\circ} \mathrm{C}$ for $30 \mathrm{~s}$.

\section{FISH}

FISH was performed on interphase nuclei using the "ThermoBrite" instrument, which is designed to programmable temperature, humidity, denaturation, and hybridization steps, and according to the protocol of the commercial probes (Vysis; Abbot Laboratories, Abbott Park, IL, USA), (ref:. 08L55-020). Briefly, we prepared the specimen target by immersing the slides in denaturing solution at $73^{\circ} \mathrm{C}$ for 2 min and dehydrated the slides for $1 \mathrm{~min}$ in 70,85 , and $100 \%$ ethanol. Next, we prepared the probe mixture as described in the commercial protocol before hybridizing. We applied $10 \mu \mathrm{L}$ mixture onto the slide and denatured the components in the Thermobrite. The slides were washed in $2 \mathrm{X} \mathrm{SSC} / 0.3 \% \mathrm{NP}-40$ solution at $73^{\circ} \mathrm{C}$ for $2 \mathrm{~min}$ and air-dried in the dark, after which $10 \mu \mathrm{L}$ counterstain was applied.

\section{RESULTS}

\section{Conventional RT-PCR and FISH}

Twenty patients diagnosed with CML were submitted to imatinib treatment for different periods and analyzed by conventional PCR to monitor $B C R-A B L$ gene expression, and therefore the presence of CML cells. Conventional RT-PCR was performed using different primers to detect fragments of 413 and $342 \mathrm{bp}$, which indicated the presence of the b3a2 
and b2a 2 major breakpoint cluster region $(\mathrm{Mbcr}$ ) variants, respectively (Figure 1). Ten of the 20 samples analyzed expressed the $B C R-A B L$ gene even after imatinib treatment and were considered to be positive samples. However, in the other 10 patient samples analyzed, no expression of $B C R-A B L$ was detected; therefore, these samples were considered to be $B C R$ $A B L$-negative (Table 1). Thus, these patients were considered to be cleared from CML cells. However, while all positive samples were confirmed to be positive according to FISH analysis, 5 of the 10 negative samples were identified as positive by FISH and still contained the $B C R-A B L$ gene (Table 1, samples S12, S14, S15, S16, and S18).

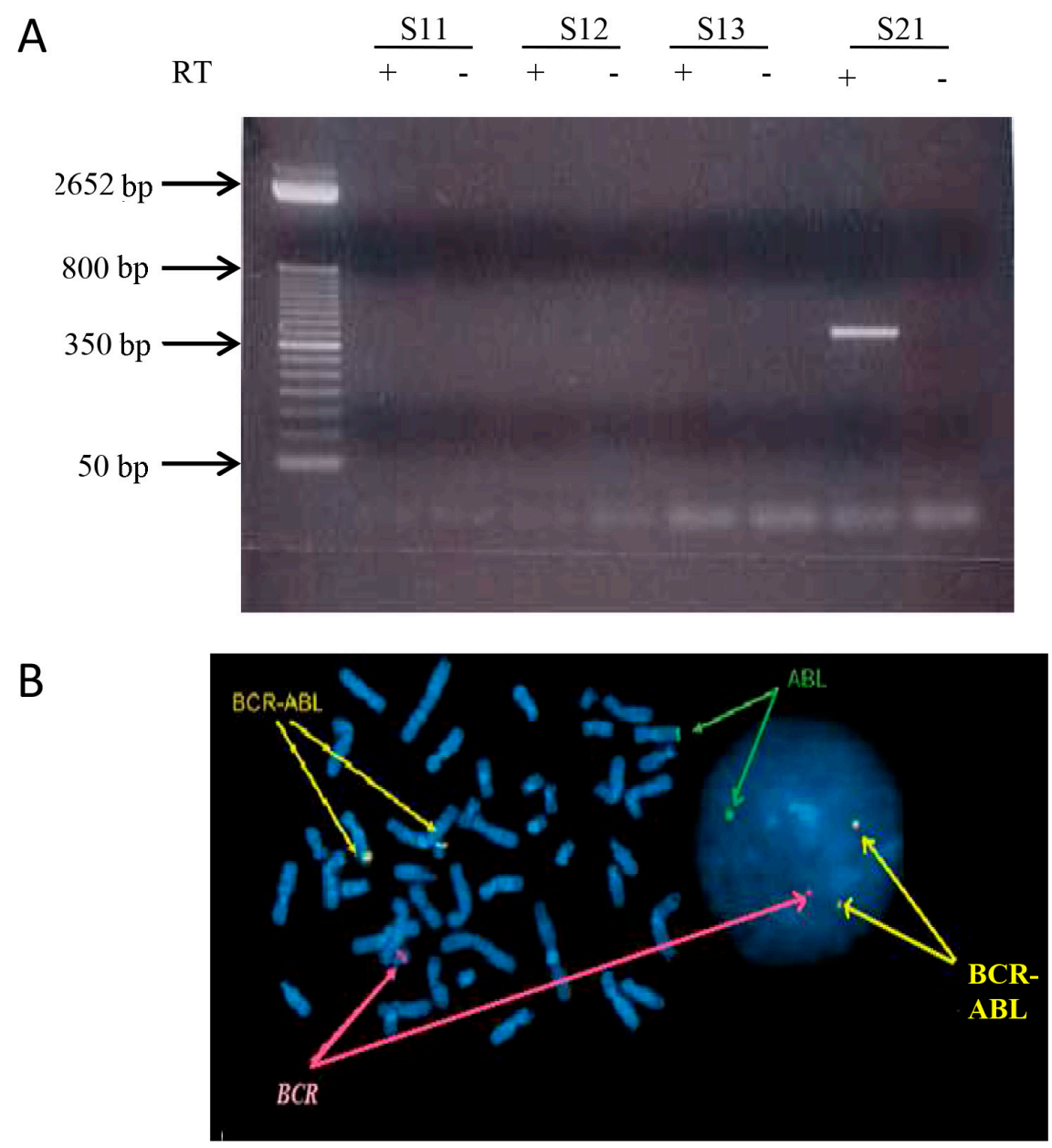

Figure 1. Detection of BCR-ABL fusion gene by conventional PCR and FISH. (A) RT-PCR was performed with different primers that could detect fragments of 413 and $342 \mathrm{bp}$ which are indicative of the presence of the b3a2 and b2a2 Mbcr variants respectively. The PCR product was loaded on an agarose gel and stained with ethidium bromide. An example of a sample positive for b3a2 Mbcr is represented (Sample 21 in Table 1). The ladder size is indicated on the left side. Only examples of positive and negative samples are indicated in this gel (B) Example of a FISH analysis performed on sample S12 (Table 1) positive for BCR-ABL fusion gene. ABL, BCR, and BCR-ABL gene are indicated by different arrows. 
Table 1. Summary of results obtained with conventional PCR, FISH, and real-time quantitative PCR for all samples analyzed.

\begin{tabular}{|c|c|c|c|c|}
\hline Names & Duration of treatment & Conventional RT-PCR & FISH & qRT-PCR \\
\hline S1 & Healthy & Negative $M$ and $m B C R$ & Negative $M$ and $m B C R$ & Negative $M$ and $m B C R$ \\
\hline S2 & Healthy & Negative $M$ and $m B C R$ & Negative $M$ and $m B C R$ & Negative $M$ and $m B C R$ \\
\hline S3 & Healthy & Negative $M$ and $m B C R$ & Negative $M$ and $m B C R$ & Negative $M$ and $m B C R$ \\
\hline S4 & Healthy & Negative $M$ and $m B C R$ & Negative $M$ and $m B C R$ & Negative $M$ and $m B C R$ \\
\hline S5 & Healthy & Negative $M$ and $m B C R$ & Negative $M$ and $m B C R$ & Negative $M$ and $m B C R$ \\
\hline S6 & Healthy & Negative $M$ and $m B C R$ & Negative $M$ and $m B C R$ & Negative $M$ and $m B C R$ \\
\hline S7 & Healthy & Negative $M$ and $m B C R$ & Negative $M$ and $m B C R$ & Negative $M$ and $m B C R$ \\
\hline S8 & Healthy & Negative $M$ and $m B C R$ & Negative $M$ and $m B C R$ & Negative $M$ and $m B C R$ \\
\hline S9 & Healthy & Negative $M$ and $m B C R$ & Negative $M$ and $m B C R$ & Negative $M$ and $m B C R$ \\
\hline S10 & Healthy & Negative $M$ and $m B C R$ & Negative $M$ and $m B C R$ & Negative $M$ and $m B C R$ \\
\hline S11 & 2 years & Negative $M$ and $m B C R$ & Negative $M$ and $m B C R$ & Negative $M$ and $m B C R$ \\
\hline S12 & 1 year & Negative $M$ and $m B C R$ & Positive $\mathrm{Mbcr}$ & Positive $M b 3 a 2$ \\
\hline S13 & 1 year & Negative $M$ and $m B C R$ & Negative $M$ and $m B C R$ & Negative $M$ and $m B C R$ \\
\hline S14 & 3 years & Negative $M$ and $m B C R$ & Positive $\mathrm{Mbcr}$ & Positive $M b 3 a 2$ \\
\hline S15 & 3 years & Negative $M$ and $m B C R$ & Positive $\mathrm{Mbcr}$ & Positive $M b 3 a 2$ \\
\hline S16 & 1 year & Negative $M$ and $m B C R$ & Positive $\mathrm{Mbcr}$ & Positive $M b 3 a 2$ \\
\hline S17 & 2 years & Negative $M$ and $m B C R$ & Negative $M$ and $m B C R$ & Negative $M$ and $m B C R$ \\
\hline S18 & 1 year & Negative $M$ and $m B C R$ & Positive $\mathrm{Mbcr}$ & Positive $M b 3 a 2$ \\
\hline S19 & 1 year & Negative $M$ and $m B C R$ & Negative $M$ and $m B C R$ & Negative $M$ and $m B C R$ \\
\hline S20 & 2 years & Negative $M$ and $m B C R$ & Negative $M$ and $m B C R$ & Negative $M$ and $m B C R$ \\
\hline S21 & 2 years & Positive $M b 3 a 2$ & Positive $\mathrm{Mbcr}$ & Positive $M b 3 a 2$ \\
\hline S22 & 3 years & Positive $M b 3 a 2$ & Positive $\mathrm{Mbcr}$ & Positive $M b 3 a 2$ \\
\hline S23 & 6 months & Positive $M b 2 a 2$ & Positive $\mathrm{Mbcr}$ & Positive $M b 2 a 2$ \\
\hline S24 & 3 months & Positive $M b 2 a 2$ & Positive $\mathrm{Mbcr}$ & Positive $M b 2 a 2$ \\
\hline S25 & 9 months & Positive $M b 3 a 2$ & Positive $\mathrm{Mbcr}$ & Positive $M b 3 a 2$ \\
\hline S26 & Not treated yet & Positive $M b 3 a 2$ & Positive $\mathrm{Mbcr}$ & Positive $M b 3 a 2$ \\
\hline S27 & 12 months & Positive $M b 2 a 2$ & Positive $\mathrm{Mbcr}$ & Positive $M b 2 a 2$ \\
\hline S28 & 12 months & Positive $M b 3 a 2$ & Positive $\mathrm{Mbcr}$ & Positive $M b 3 a 2$ \\
\hline S29 & 12 months & Positive $M b 2 a 2$ & Positive $\mathrm{Mbcr}$ & Positive $M b 2 a 2$ \\
\hline S30 & 6 months & Positive $M b 3 a 2$ & Positive $M b 2 a 2$ & Positive $M b 3 a 2$ \\
\hline
\end{tabular}

RT-PCR: reverse transcription-polymerase chain reaction; FISH: Fluorescence in situ hybridization; Mbcr: major breakpoint cluster region; Mbcr: minor breakpoint cluster region.

\section{Real-time PCR}

To evaluate the real-time qPCR method developed in our laboratory and to determine whether the same results were obtained with conventional RT-PCR and FISH, we evaluated $B C R-A B L$ gene expression in all 20 samples using a specific set of primers and probes designed in-house. The specificity and sensitivity of our primers and probes were assessed using mRNA extracted from in vitro-cultured K562 and HL60 cells, which are BCR-ABLpositive and $B C R-A B L$-negative leukemic cell lines, respectively. Indeed, while stable and clear $B C R-A B L$ expression was detected in the K562 cell line, no $B C R-A B L$ was detected using the $B C R-A B L$-negative HL60 cells (Figure 2A). In contrast, both cell lines expressed the $A B L$ gene used as a reference gene in this study (Figure 2A). Furthermore, to measure the efficiency of the PCR under our conditions, a standard curve was generated using a serial dilution of a known cDNA concentration from the K562 cell line and a plasmid vector. This vector, $B C R-A B L / A B L$, contained fragments from both the $B C R-A B L$ and $A B L$ genes and encompassed the region recognized and amplified by our set of primers and probes. As shown in Figure 2, qPCR efficiency under our conditions was greater than $95 \%$ which is in accordance with the required international in vitro diagnostic standard efficiency for a real-time PCR with high performance (Livak and Schmittgen, 2001) (Figure 2B-E). In addition, the $\mathrm{R}^{2}$ factor is used to measure the performance of a real-time PCR reaction. $\mathrm{R}^{2}$ is a correlation 
factor indicating how well the data fits the model and how well the data fits a straight line. This factor is influenced by pipetting accuracy and assay range. The $\mathrm{R}^{2}$ value must always be higher than or equal to 0.985 for the results to be reliable (Livak and Schmittgen, 2001). Our $\mathrm{R}^{2}$ value was consistently $\geq 0.99$, indicating a high accuracy of the qPCR under our experimental conditions (Figure 2B-E).
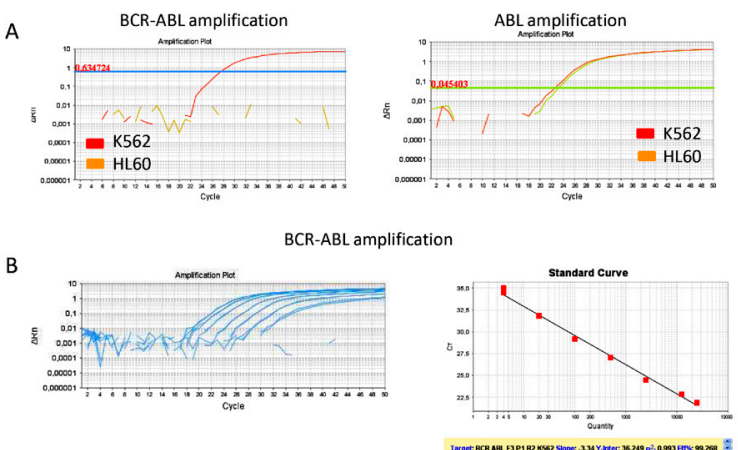

C
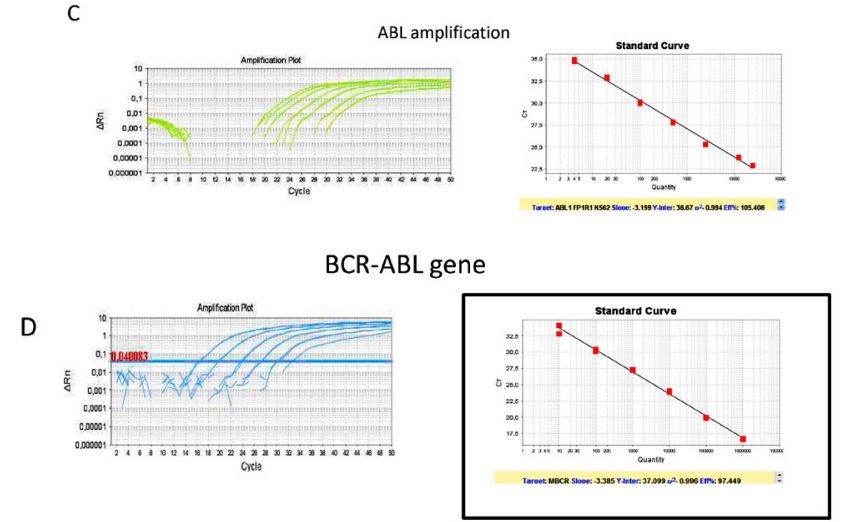

$\mathrm{ABL}$ gene

E
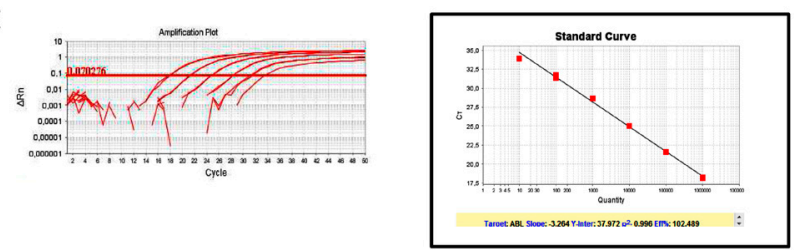

Figure 2. Detection of $B C R-A B L$ fusion gene by real time qPCR. (A) qPCR was performed with the same primer set used for the conventional PCR in addition to a TaqMan probe and amplification of the $B C R-A B L$ and $A B L$ genes was followed with cDNA prepared from both K562 cell line (BCR-ABL positive) and HL60 (BCR-ABL negative). (B) A standard curve was generated from serial dilution of cDNA prepared from K562 cell line amplifying an amplicon from the $B C R-A B L$ gene. (C) A standard curve was generated from serial dilution of cDNA prepared from K562 cell line amplifying an amplicon from the $A B L$ gene. (D) Standard curves prepared from serial dilution of plasmid vectors containing fragment of $B C R-A B L$. (E) Standard curves prepared from serial dilution of plasmid vectors containing fragment of the $A B L$ gene. Both the efficiency and $\mathrm{R}^{2}$ values for the qPCR are shown. 


\section{Comparison of real-time PCR, FISH, and conventional PCR}

After establishing and validating the sensitivity, specificity, and performance of the PCR under our conditions, we assessed $B C R-A B L$ gene expression in 20 patient samples subjected to imatinib treatment. In addition, we used both $B C R-A B L$-negative leukemic HL60 cells and normal PBMCs collected from disease-free subjects as negative controls. We generated a standard curve using serial dilution of the $B C R-A B L / A B L$ vector with a known plasmid copy number (Figure 3A). This standard curve allowed not only determination of the PCR efficiency and $\mathrm{R}^{2}$, but also extrapolation of the copy number of both $B C R-A B L$ and $A B L$ transcript in the patient samples. The ratio of $B C R-A B L$ to $A B L$ copy number was determined, compared to the threshold set from the negative control cell lines, and used to evaluate $B C R-A B L$ gene expression. The results are summarized in Table 1 and Figure 3B. All samples that were positive for $B C R-A B L$ expression according to the results of conventional PCR were also positive with real-time PCR, indicating high concordance between the 2 methods (Table 1 and Figure 3B). However, in contrast with conventional PCR, 5 of 10 negative samples were positive for $B C R-A B L$ expression by qPCR (Table $1, \mathrm{~S} 12, \mathrm{~S} 14, \mathrm{~S} 15, \mathrm{~S} 16$, and $\mathrm{S} 18$ ). The $B C R-A B L$ to $A B L$ ratios were clearly higher than the set threshold and sometimes higher than the ratio observed for the positive samples (Table 1 and Figure 3B). In addition, all samples were confirmed to be positive using FISH analysis (Table 1). This suggests that these samples were still positive for $B C R-A B L$ gene expression and that $\mathrm{qPCR}$ is more sensitive and potent than conventional $\mathrm{PCR}$, and may be used to replace FISH.
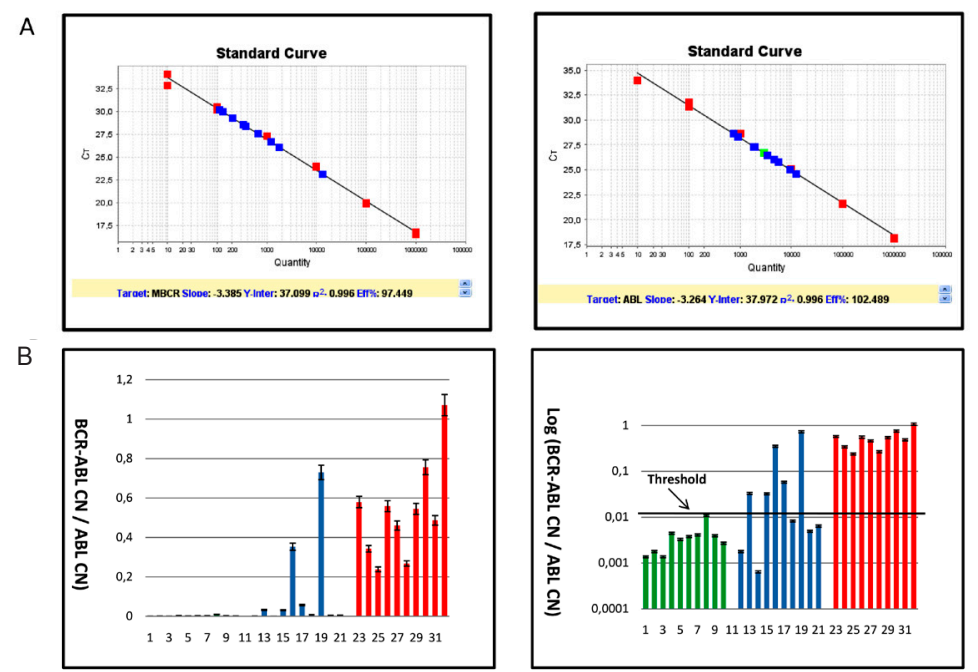

Figure 3. Comparison between qPCR, FISH, and conventional PCR results. (A) Extrapolation of the samples analyzed (only some samples are represented) using standard curve generated as in Figure 2C. Positive and negative samples are indicated by blue spots. (B) Chart representation of the value of copy number ratio BCR$\mathrm{ABL} / \mathrm{ABL}$ of all the samples analyzed. Healthy samples, samples determined negative by conventional PCR, and samples determined positive by conventional PCR, FISH, and qPCR are respectively indicated by green, blue, and red charts. A solid black line indicates the threshold of positivity. In the chart on the right the $\mathrm{Y}$ axis is represented with a logarithmic scale. The value presented for each sample is the mean of three different experiments and the standard deviation has been calculated using the Excel software. 


\section{DISCUSSION}

The introduction of imatinib in 1998 significantly declined the treatment-related mortality by $30 \%$ for CML patients exposed to therapy compared with a control group of CML patients who underwent stem cell transplantation prior to the availability of imatinib (Savage and Antman, 2002; Capdeville et al., 2002). The presence of the $B C R-A B L$ gene and its products are considered to be a surrogate for disease activity, and its clearance is considered to be a prerequisite for developing a cure. Therefore, the methods used to monitor CML are very important as they will dictate whether a patient must continue imatinib treatment or is considered to be CML-free.

In this study, we investigated the usefulness of real-time RT-qPCR to quantify $B C R$ $A B L$ transcript levels with an accuracy that allows prediction of cytogenetic relapse by monitoring $B C R-A B L$ transcript levels following imatinib treatment of 20 Moroccan CML patients. We compared conventional RT-PCR, FISH, and real-time RT-qPCR to determine their efficiencies for detecting and quantifying $B C R-A B L$ transcript levels. Our results showed that samples that were $B C R-A B L$-positive by both conventional PCR and FISH were also positive according to real-time RT-qPCR (Table 1). However, 5 of the samples determined to be $B C R$ $A B L$-negative by conventional PCR expressed the $B C R-A B L$ gene as detected by real-time RTqPCR. This constituted $25 \%$ of the samples analyzed and clearly indicated that conventional RT-PCR was less sensitive than real-time RT-qPCR in detecting the $B C R-A B L$ transcript. However, the results of FISH confirmed the results of RT-qPCR, as the same negative samples shown to be positive by RT-qPCR were determined to be positive by FISH (Table 1). This indicated high concordance between FISH and RT-qPCR for detecting the $B C R-A B L$ transcript.

Cytogenetic analysis remains the "gold standard" for diagnosing and following up CML. Although this method presents some advantages, its low sensitivity (less than 10\%) and the requirement for bone marrow samples in metaphase limits its use for monitoring of CML. The other method used is FISH, which is faster and more accurate than cytogenetic methods (Buño et al., 1998; Wang et al., 2001). Unlike cytogenetic methods, which require dividing of metaphase cells, FISH can be performed on interphase nuclei in both PBMCs and bone marrow cells. However, the sensitivity of interphase FISH is limited by the frequency with which $B C R$ and $A B L$ signals randomly co-localize in normal cells (Chase et al., 1997). In practice, the limit of detection of CML cells depends in part on which probes are used, the size of the nucleus, the precise portion of the breakpoint within the $A B L$ gene, and the criteria used to define co-localization. Moreover, FISH is technically difficult to set up, time-consuming, and very expensive. Thus, a new easy molecular method that can accurately measure $B C R-A B L$ transcript levels and monitor CML disease, mainly detection of the MRD following treatment, is necessary. Currently, the most sensitive method for detecting and monitoring $B C R-A B L$ expression is RT-PCR (Radich et al., 1995; Colleoni et al., 2000). Similarly to FISH, RT-PCR does not require a cell to divide, and can therefore also be used for PBMCs. Indeed, it has been shown that qualitative results obtained by RT-PCR using PBMCs are in high concordance with those obtained using bone marrow cells (Verschraegen et al., 1995). In addition, RT-PCR can be used to detect $1 B C R-A B L$-positive cell in 100-1000 normal cells in $1 \mu \mathrm{g}$ RNA used (Colleoni et al., 2000). This highlights the high sensitivity of this method for detecting the $B C R-A B L$ transcript (Colleoni et al., 2000). In the current study, we compared the sensitivity of both conventional RT-PCR and real-time RT-qPCR in detecting $B C R-A B L$ gene expression. Using qPCR, a standard curve was generated from a serial dilution of known copy number of a plasmid vector containing fragments of both the $B C R-A B L$ and $A B L$ genes encompass- 
ing the region recognized and amplified by our set of primers and probes. Using this standard curve, we accurately determined the copy number of the $B C R-A B L$ or $A B L$ transcripts using the cycle threshold $(\mathrm{Ct})$ value of the qPCR-analyzed samples. In addition, the standard curve allowed for determination of both qPCR efficiency and the $\mathrm{R}^{2}$ correlation factor, which are essential for monitoring the performance of the qPCR test. The qPCR efficiency was consistently above $95 \%$, demonstrating that in our conditions; the qPCR method is very efficient and can be used to detect very low levels of $B C R-A B L$ gene transcripts. Furthermore, the accuracy of our qPCR-based test was high, as the $\mathrm{R}^{2}$ factor was always superior to 0.99 , the minimum value required for a highly accurate $\mathrm{qPCR}$-based test. Therefore, $B C R-A B L$ transcript copy numbers from patient samples were very efficiently and accurately detected using the qPCRbased test. We set a $B C R-A B L / A B L$ copy number ratio threshold of detection using the $B C R$ $A B L$-negative cells. Thus, in our experiment, any value above this threshold indicated positive $B C R-A B L$ transcript expression. As shown in Figure 3B, 5 of the 10 patients determined to be $B C R-A B L$-negative by conventional PCR had a $B C R-A B L / A B L$ copy number ratio above the set threshold. This indicates that these patients still expressed the $B C R-A B L$ gene and therefore should continue the imatinib treatment.

\section{CONCLUSIONS}

In conclusion, our results demonstrate that the cost-effective method qPCR is potent and sensitive for determining $B C R-A B L$ expression in Moroccan CML-affected patients and should be used locally to monitor disease progression, particularly MRD following imatinib treatment. Nevertheless, additional CML patient blood samples should be analyzed to confirm these data.

\section{ACKNOWLEDGMENTS}

We thank all patients that have kindly agreed to provide us with the samples analyzed here. We are also very grateful to Prof. Veronica Caroll (University of London) for her critical reading of our manuscript. We are also indebted to all members of the medical biotechnology laboratory for their help in performing this research.

\section{REFERENCES}

Buño I, Wyatt WA, Zinsmeister AR, Dietz-Band J, et al. (1998). A special fluorescent in situ hybridization technique to study peripheral blood and assess the effectiveness of interferon therapy in chronic myeloid leukemia. Blood 92: 2315-2321.

Capdeville R, Buchdunger E, Zimmermann J and Matter A (2002). Glivec (STI571, imatinib), a rationally developed targeted anticancer drug. Nat. Rev. Drug Discov. 1: 493-502.

Chase A, Grand F, Zhang JG, Blackett N, et al. (1997). Factors influencing the false positive and negative rates of BCRABL fluorescence in situ hybridization. Genes Chromosomes Cancer 18: 246-253.

Colleoni GW, Jhanwar SC, Ladanyi M and Chen B (2000). Comparison of a multiplex reverse transcriptase-polymerase chain reaction for BCR-ABL to fluorescence in situ hybridization, Southern blotting, and conventional cytogenetics in the monitoring of patients with Ph1-positive leukemias. Diagn. Mol. Pathol. 9: 203-209.

Daley GQ, Van Etten RA and Baltimore D (1990). Induction of chronic myelogenous leukemia in mice by the P210bcr/ abl gene of the Philadelphia chromosome. Science 247: 824-830.

Druker BJ, Talpaz M, Resta DJ, Peng B, et al. (2001). Efficacy and safety of a specific inhibitor of the BCR-ABL tyrosine kinase in chronic myeloid leukemia. N. Engl. J. Med 344: 1031-1037.

Faderl S, Talpaz M, Estrov Z, O'Brien S, et al. (1999). The biology of chronic myeloid leukemia. N. Engl. J. Med 341: 
164-172.

Heisterkamp N, Stam K, Groffen J, de Klein A, et al. (1985). Structural organization of the bcr gene and its role in the Ph' translocation. Nature 315: 758-761.

Hughes TP, Kaeda J, Branford S, Rudzki Z, et al. (2003). Frequency of major molecular responses to Imatinib or interferon alfa plus Cytarabine in newly diagnosed chronic myeloid leukaemia. N. Engl. J. Med. 349: 1423-1432.

Kantarjian H, Sawyers C, Hochhaus A, Guilhot F, et al. (2002). Hematologic and cytogenetic responses to imatinib mesylate in chronic myelogenous leukemia. N. Engl. J. Med 346: 645-652.

Livak KJ and SchmittgenTD (2001). Analysis of relative gene expression data using real-time quantitative PCR and the 2(-Delta Delta C(T)) Method. Methods 25: 402-408.

Lugo TG, Pendergast AM, Muller AJ and Witte ON (1990). Tyrosine kinase activity and transformation potency of bcr-abl oncogene products. Science 247: 1079-1082.

Lyseng-Williamson K and Jarvis B (2001). Imatinib. Drugs 61: 1765-1776.

Radich JP, Gehly G, Gooley T, Bryant E, et al. (1995). Polymerase chain reaction detection of the BCR-ABL fusion transcript after allogeneic marrow transplantation for chronic myeloid leukemia: results and implications in 346 patients. Blood 85: 2632-2638.

Rowley JD (1973). Letter: a new consistent chromosomal abnormality in chronic myelogenous leukemia identified by quinacrine fluorescence and Giemsa staining. Nature 243: 290-293.

Sattler M and Griffin JD (2003). Molecular mechanisms of transformation by the BCR-ABL oncogene. Semin. Hematol. 40: 4-10.

Savage DG and Antman KH (2002). Imatinib mesylate-a new oral targeted therapy. N. Engl. J. Med. 346: 683-693.

Sawyers CL (1999). Chronic myeloid leukemia. N. Engl. J. Med. 340: 1330-1340.

Sillaber C, Mayerhofer M, Agis H, Sagaster V, et al. (2003). Chronic myeloid leukemia: pathophysiology, diagnostic parameters, and current treatment concepts. Wien Klin. Wochenschr 115: 485-504.

Verschraegen CF, Talpaz M, Hirsch-Ginsberg CF, Pherwani R, et al. (1995). Quantification of the breakpoint cluster region rearrangement for clinical monitoring in Philadelphia chromosome positive chronic myeloid leukemia. Blood 85: 2705-2710.

Wang YL, Bagg A, Pear W, Nowell PC, et al. (2001). Chronic myeloid leukemia: laboratory diagnosis and monitoring. Genes Chromosomes Cancer 32: 97-111. 